\title{
Mediating Mechanisms in Cognitive Behavioral Therapy for Childhood OCD: The Role of Dysfunctional Beliefs
}

\author{
L. H. Wolters ${ }^{1,2}$ - P. J. M. Prins ${ }^{3}$ - G. J. A. Garst ${ }^{3}$ - S. M. Hogendoorn ${ }^{2} \cdot$ F. Boer ${ }^{1} \cdot$ L. Vervoort ${ }^{4}$ - E. de Haan ${ }^{2}$
}

Published online: 21 July 2018

(c) The Author(s) 2018

\begin{abstract}
Reframing cognitions is assumed to play an important role in treatment for obsessive-compulsive disorder (OCD). However, there hardly is any empirical support for this assumption, especially for children. The aim of this study was to examine if changing dysfunctional beliefs is a mediating mechanism of cognitive behavioral therapy (CBT) for childhood OCD. Fiftyeight children (8-18 years) with OCD received CBT. Dysfunctional beliefs (OBQ-CV) and OCD severity (CY-BOCS) were measured pre-treatment, mid-treatment, post-treatment, and at 16-week follow-up. Results showed that OCD severity and dysfunctional beliefs decreased during CBT. Changes in severity predicted changes in beliefs within the same time interval. Our results did not support the hypothesis that changing dysfunctional beliefs mediates treatment effect. Future studies are needed to replicate these findings and shed more light on the role of explicit and implicit cognitions in treatment for childhood OCD.
\end{abstract}

Keywords Pediatric obsessive compulsive disorder · Cognitive behavioral therapy $\cdot$ Mediator of treatment $\cdot$ Obsessive beliefs · Cognitive theory

\section{Introduction}

The effectiveness of cognitive behavioral therapy (CBT) for childhood obsessive compulsive disorder (OCD) has been well established [1-5]. However, average symptom reduction is limited and almost half of the patients still have considerable complaints after standard treatment [6-8]. The way CBT is applied may not always be optimally effective [9-11].

L. H. Wolters

L.H.Wolters@amc.uva.nl

1 Department of Child and Adolescent Psychiatry, Academic Medical Center, Meibergdreef 5, 1105 AZ Amsterdam, The Netherlands

2 Academic Center for Child and Adolescent Psychiatry, De Bascule, Meibergdreef 5, 1105 AZ Amsterdam, The Netherlands

3 Faculty of Social and Behavioural Sciences, University of Amsterdam, Nieuwe Achtergracht 127, 1018 WS Amsterdam, The Netherlands

4 Department of developmental, personality and social psychology, Ghent University, Henri Dunantlaan 2, 9000 Ghent, Belgium
CBT for OCD intends to change behavior (compulsions) and cognitions (obsessions). This is mostly done by a combination of exposure plus response prevention (ERP), and cognitive therapy (CT) [1]. Despite their common basis in the learning theory, distinct mechanisms leading to symptom reduction are assumed in ERP and in CT. From a behavioral perspective it is assumed that a behavioral change through exposure to feared situations is the primary process leading to essential corrective learning experiences. For this reason, behaviorists advocate ERP as the core of treatment [11-13]. In the cognitive model, it is assumed that the optimal way to corrective learning is through explicitly reframing dysfunctional beliefs. Therefore, in CT cognitive restructuring is advocated as the core of treatment $[14,15]$.

In line with the cognitive model, a relation between dysfunctional beliefs and obsessive-compulsive (OC) symptoms has been found in several child studies [16-25]. However, other studies yielded equivocal evidence for the cognitive model in childhood OCD [26-29]. In two studies it has been tested if inflated responsibility beliefs affected OC symptoms in children based on an experimental design. These studies yielded mixed results [24, 26]. Taken together, despite a well-developed theoretical base for a key role of 
dysfunctional beliefs in (childhood) OCD, the evidence is equivocal.

Nevertheless, cognitive models have strongly influenced treatment for OCD. Almost all treatment packages for childhood OCD contain some type of cognitive interventions [1, 2, 30, 31]. Full cognitive treatment protocols are developed even for children [32], and in combined treatment packages it is not unusual to start with a focus on cognitive interventions, followed by ERP. Efficacy of behavioral as well as cognitive oriented treatment protocols have been demonstrated [1,33]. However, a favorable effect of ERP over CT has been reported [34], and the addition of cognitive interventions to ERP did not always result in more effective treatment packages $[9,35]$. Overall, critiques on cognitive models are arising [35].

At present, the core of CBT is still unknown. Is explicitly changing cognitions the main mechanism through which symptom reduction is achieved, or would it be more effective to primarily focus on ERP?

A better understanding of the mechanisms of change in CBT may contribute to better treatment $[1,33]$. Identification of these mechanisms can help to improve treatment because effective treatment components can be added or strengthened, and ineffective components can be removed [36]. However, mechanisms of change are rarely studied in treatments for childhood OCD.

Studying change mechanisms in treatment requires a formal test of statistical mediation. Mediation refers to the process through which change occurs. A change in the proposed mediator variable should precede a change in outcome. Consequently, a repeated measurements design is needed to demonstrate mediation [37]. Unfortunately, most treatment studies traditionally rely on pre-post test designs. An exception is a small pilot study [38]. In this study it was examined if changes in dysfunctional cognitions were associated with treatment effect in CT for pediatric OCD. Six adolescents with OCD (12-17 years, $M=14.3)$ reported responsibility beliefs and OCD symptoms at every treatment session. It was found that decreases in responsibility beliefs were associated with decreases in OCD symptoms, but the direction of this relation remained unclear, leaving the question of mediation unanswered [38].

Repeated measurements designs have been used slightly more often in adult OCD studies, with equivocal results. Whereas the findings of some studies provided some support for the cognitive model $[39,40]$, findings of other studies did not [34, 41-44], or were inconclusive [45, 46]. Most studies are based on small samples, and differences across studies in design and statistical analyses make it hard to draw clear conclusions. A preliminary observation is that most studies, including those with the largest sample sizes and based on thorough statistical analyses [34, 43, 44], did not support the cognitive mediation hypothesis.
Given the mixed evidence with regard to the potential role of dysfunctional beliefs in the treatment of childhood OCD, the aim of the present study was to further examine if changing dysfunctional cognitions is a mediator of treatment outcome in CBT for children and adolescents with OCD. It was examined whether changes in dysfunctional beliefs preceded changes in OCD severity, whether they were a consequence of changes in OCD severity, or whether this relation was bidirectional. Based on cognitive models it was our hypothesis that cognitive changes precede changes in OCD severity.

\section{Methods}

\section{Design and Procedure}

The present study was part of a trial intended to study psychological and neurobiological processes, non-response, and mediators of treatment outcome in childhood OCD. The trial was approved by the Medical Ethics Committee of the Academic Medical Center (MEC 06/053). ${ }^{1}$ The design and procedure of the study has been previously described in detail [8]. Participants were children and adolescents (8-18 years) who were referred for treatment for OCD to one of our treatment centers in the Netherlands. Inclusion criteria were a primary diagnosis of OCD according to The Diagnostic and Statistical Manual of Mental Disorders (4th ed., text revision; DSM-IV-TR) criteria, OCD complaints for at least 6 months, and a minimum score of 16 on the Children's Yale-Brown Obsessive Compulsive Scale (CYBOCS). Exclusion criteria were medication for OCD (SSRI, tricylic antidepressants or antipsychotic medication), CBT for OCD during the past 6 months, IQ below 80, and psychosis. Informed consent was obtained from all individual participants included in the study. Next, participants were randomly assigned to CBT or waitlist followed by CBT. CBT did not differ across conditions.

Assessments were conducted pre-treatment (T1), midtreatment (T2; session 8), post-treatment (T3; session 16) and at 16 weeks follow-up (T4). During the assessments, the Children's Yale-Brown Obsessive Compulsive Scale (CY-BOCS; see below) and the Obsessive Belief Questionnaire-Child Version (OBQ-CV; see below) were administrated. Additional assessments and measures for the purpose of the full trial are not described here. Participants received a small compensation for their efforts after completing the

\footnotetext{
${ }^{1}$ Clinical trial registration information: Information processing, neuropsychological, and neurobiological processes in pediatric obsessive-compulsive disorder; http://www.controlled-trials.com/ISRCT N07851536;NTR 717, ISRCTN07851536.
} 
Fig. 1 Flow chart

\begin{tabular}{|c|c|}
\hline \multicolumn{2}{|c|}{ Assessed for eligibility $(N=73)$} \\
\hline & $\begin{array}{l}\text { Excluded }(n=12) \\
\text { Not meeting inclusion criteria }(n=7) \\
\text { Declined to participate }(n=1) \\
\text { Decided no treatment }(n=1) \\
\text { Other/reason not reported }(n=3)\end{array}$ \\
\hline \multicolumn{2}{|c|}{ Included $(N=61)$} \\
\hline \multicolumn{2}{|l|}{$\begin{array}{l}\text { T1: pre-CBT }(N=58) \\
\text { Lost to T1 }(n=3) \\
\text { Not meeting inclusion criteria anymore }(n=2) \\
\text { Not able to come due to family problems }(n=1) \\
\text { Missing data } \\
\text { OBQ }(n=4)\end{array}$} \\
\hline \multicolumn{2}{|l|}{$\begin{array}{l}\text { T2: } \text { mid-CBT }(N=53) \\
\text { Lost to T2 }(n=5) \\
\text { Discontinued CBT: no complaints anymore }(n=1) \\
\text { Failed to turn up at treatment appointments }(n=1) \\
\text { Refused CBT }(n=1) \\
\text { Medication and/or inpatient treatment }(n=2)\end{array}$} \\
\hline \multicolumn{2}{|c|}{$\begin{array}{l}\text { T3: post-CBT ( } N=48) \\
\text { Lost to T3 }(n=5) \\
\text { Discontinued CBT, not able to come due to family reasons }(n=1) \\
\text { Failed to turn up at treatment appointments }(n=1) \\
\text { Switch of treatment focus }(n=2) \\
\text { Medication and inpatient treatment }(n=1) \\
\text { Missing data }(n=4) \\
\text { Discontinued CBT, no OCD anymore }(n=2) \text { [back at T4] } \\
\text { OBQ }(n=2)\end{array}$} \\
\hline \multicolumn{2}{|c|}{$\begin{array}{l}\text { T4: 16-week follow-up }(N=43) \\
\text { Lost to T4 }(n=5) \\
\text { Study drop out (postponed from study, CBT continued) }(n=1) \\
\text { Inpatient treatment }(n=2) \\
\text { Organizational reasons }(n=2) \\
\text { Missing data }(n=1) \\
\text { OBQ }(n=1)\end{array}$} \\
\hline
\end{tabular}

assessments (i.e., a gift voucher with a value of 10 Euro for each assessment).

\section{Participants}

Between January 2007 and June 2010, 73 children were screened for eligibility in the study and 61 children (84\%) were included. Three children dropped out before the pretreatment assessment (T1): two did not meet the inclusion criterion anymore, and one child was not able to visit the clinic due to family circumstances. Finally, 58 children were included. Forty-six of the 58 children (79\%) completed the CBT. Reasons for drop out were: early termination of treatment because complaints were in remission $(n=3)$, OCD symptoms were in remission before session 16 and treatment switched to other problems $(n=2)$, patients were unable or unwilling to visit the clinic $(n=4)$, referral to inpatient treatment for OCD and/or co-morbid problems $(n=2)$, and addition of medication $(n=1)$. Forty-eight children completed the post-CBT (T3) assessment, and 43 children completed the 16-week follow-up (T4) assessment (see Fig. 1 for the flow chart). The trial was ended because the intended number of participants was reached. No adverse events were reported.

Demographic and clinical characteristics of the sample have been previously described in more detail [8], and are summarized in Table 1.

\section{Treatment}

Treatment consisted of 16 weekly sessions of manualized individual CBT described in the Dutch treatment manual 
Table 1 Baseline characteristics of participants $(N=58)$

\begin{tabular}{lll}
\hline Age & $M(\mathrm{SD})$ & $12.8(2.6)$ \\
Gender & Boys & $24(41.4 \%)$ \\
& Girls & $34(58.6 \%)$ \\
Cultural background & Dutch & $46(79.3 \%)$ \\
& Non-western & $3(5.2 \%)$ \\
& Other/combined & $4(6.9 \%)$ \\
CY-BOCS & Missing & $5(8.6 \%)$ \\
ADIS & $M(\mathrm{SD})$ & $24.8(4.1)$ \\
& range & $16-35$ \\
& Any co-morbidity & $38(65.5 \%)$ \\
& Anxiety disorder & $31(53.4 \%)$ \\
& Mood disorder & $9(15.5 \%)$ \\
& ADHD/ODD & $9(15.5 \%)$ \\
\hline
\end{tabular}

'Bedwing je dwang' ('Control your OCD') [47]. The treatment involves psychoeducation, building a hierarchy of OC symptoms, exposure with response prevention (ERP), cognitive interventions (CI), and relapse prevention. ERP is introduced early in treatment (second session), followed by and combined with cognitive interventions (start at second or third session). ERP involved therapist-assisted practice during the sessions and (daily) exercises at home. Cognitive treatment contained a basic part (information about cognitions and their relation with feelings and behavior, and about the role of cognitions in OCD), and a range of additional interventions varying in complexity from simple (for example, replacing dysfunctional thoughts by thoughts that help to cope with anxiety or distress) to more sophisticated cognitive techniques (for example, probability estimates, and listing pros and cons) in order to enable therapists to tailor treatment to the individual. Guidelines for the selection of cognitive interventions, based on age, intellectual level, interest/motivation, and insight in their complaints, were provided in the manual. All participants received the basic part and one or more additional cognitive interventions during treatment. Treatment sessions lasted 45-60 min. Parents were involved in the therapy. CBT was delivered by master level clinicians certified as cognitive behavioral therapists and experienced in treating OCD in children. Therapists were trained in the protocol and attended group supervision every 2 weeks combined with optional individual supervision by the last author.

\section{Treatment Adherence}

To allow for the assessment of treatment adherence, therapists completed a report of each treatment session. Twentyfive percent of the session reports of each participant were evaluated by two independent raters. Criteria for adequate treatment adherence were: psychoeducation and building a hierarchy of OC symptoms during the first session, ERP and/or CI and homework exercises during session 2-15, and relapse prevention in the last session. For $98.5 \%$ of the sessions criteria for adequate treatment adherence were met. Raters agreed for $99 \%$ of the session reports, Cohen's kappa was 0.75 .

\section{Measures}

The Anxiety Disorder Interview Schedule for DSM-IV-Child and Parent Version (ADIS-C/P) [48, 49] is a semi-structured interview evaluating prevalence and severity of DSM-IV diagnoses of anxiety disorders, mood disorders, ADHD and disruptive disorders. The ADIS-C/P has demonstrated good to excellent test-retest and interrater reliability, and adequate concurrent validity [50,51]. A clinician severity rating (CSR, ranging from 0 to 8 ) of at least four is indicative of a diagnosis. The combined CSR was used, which means that in cases in which the child and parent interviews yield the same diagnosis, the higher of the two severity ratings was assigned. Interviews were videotaped and 19 interviews (random selection) were independently rated by two raters to examine inter-rater reliability. Cohen's kappa was 0.93 .

The Children's Yale-Brown Obsessive Compulsive Scale (CY-BOCS) [52] severity scale is a clinician-rated semistructured interview evaluating the severity of OC symptoms, and consists of an obsession and a compulsion subscale. Each subscale contains 5 items assessing frequency/ time, interference, distress, resistance, and control. Items are rated by the clinician on a 5-point scale from 0 to 4 . The total score, the sum of both subscales, ranges from 0 to 40 . A total score of 16 or more is considered as clinically significant [6]. The CY-BOCS demonstrated good reliability and adequate divergent and convergent validity [52]. Cronbach's $\alpha$ in the present study ranged from 0.81 to 0.96 . To examine inter-rater reliability, interviews were videotaped and 46 interviews (random selection) were independently rated by three raters (investigators and therapists). The intraclass correlation coefficient was 0.98 .

The Dutch version of the Obsessive Belief Questionnaire-Child Version (OBQ-CV) [28] was used. The OBQ$\mathrm{CV}$ is a self-report questionnaire about OCD-related dysfunctional beliefs, consisting of three subscales (44 items): Responsibility/Threat Estimation (RT), Perfectionism/Certainty (PC), and Importance/Control of Thoughts (ICT). Answers are scored on a five-point scale, ranging from 1 (never) to 5 (always), with higher scores indicating more obsessive beliefs. A study on psychometric properties of the Dutch version of the OBQ-CV in a community sample $(N=547 ; 8-18$ years $)$ and an OCD sample $(N=67$; 8-18 years) yielded support for its reliability and validity. Cronbach's alpha was 0.95 , and retest reliability was adequate. Confirmatory factor analyses revealed best fit for 
a four-factor model representing Perfectionism/Certainty, Importance/Control of Thoughts, Responsibility, and Threat, and a higher-order factor [28]. Cronbach's $\alpha$ (T1-T4) in the present study ranged from 0.96 to 0.98 .

\section{Statistical Analyses}

The intention-to-treat principle was used for the analyses, unless otherwise mentioned. For missing data at the level of missing items, missing values on the OBQ-CV were replaced by the individual mean of all valid items. In case of more than 5 missing items the OBQ-CV was considered as missing completely. Cases with missing measures or assessments were compared to complete cases on age, gender, pre-treatment OCD severity (CY-BOCS) and pre-treatment dysfunctional beliefs (OBQ). There were no significant differences between groups. Patterns of missing data were inspected using the Missing Value Analysis (MVA) output and Little's Missing Completely At Random (MCAR) test in SPSS. Little's MCAR test did not reach significance. We found no evidence that the missingness of data were related to any variable in this study. Missing assessments (OBQ-CV, CY-BOCS) were imputed using the expectation-maximization (EM) algorithm in LISREL version 8.8. Fifty-eight participants were included in the analyses.

\section{Effect of Treatment}

A prerequisite for a mediation analysis is an effective treatment. To test whether this condition was met, we performed a linear mixed model analysis in SPSS with time (T1-T4) as independent variable and OCD severity (CYBOCS) as dependent variable. This analysis was performed with both an unstructured covariance matrix and an autoregressive heterogeneous matrix. Fit of both models were compared using the $-2 \log$ likelihood values. As results revealed no significant difference in fit between models, preference was given to the autoregressive heterogeneous matrix as this matrix provided a more parsimonious model.

\section{Mediation Analysis}

A series of latent different score (LDS) models was used to examine if changes in dysfunctional beliefs mediated treatment outcome [53]. Analyses were conducted with LISREL version 8.8. All models were estimated using maximum likelihood.

LDS models are recommended for examining within-individual change over time in situations in which change may not be constant for each interval in the model [54]. The latent difference score (or 'gain score') variables in these models represent the difference between two successive latent true scores. Latent true score variables are composed of the preceding latent true score and an accumulation of latent changes over time (see Fig. 2). In addition, LDS models allow for examination of within-individual change as well as individual differences in the within-individual change [54, 55]. An advantage of LDS models relative to other models that could be used to examine change over time (e.g., latent growth curve (LGC) models) is that trajectories over time are free to vary, which means that there are no restrictions on the nature of change over time. In contrast to autoregressive (AR) models, changes are not restricted to residual changes. The dynamic latent difference score model [56] was also taken into consideration. However, in these models change scores are composed of a systematic trend-like component (as in LGC models), and a component explained by the previous assessments (as in AR models). Both components impose restrictions on change trajectories over time.

In conclusion, LDS models allow for testing dynamic relationships between dysfunctional beliefs and OCD severity without imposing a particular change mechanism on the data. As we had no specific hypothesis concerning the change trajectories over time of both variables, we preferred the LDS model over the Dynamic LDS Model to study the dynamic interplay between changes in dysfunctional beliefs and OCD severity.

To restrict the number of parameters to be estimated in the LDS models, we used the total scores of the OBQ-CV and the CY-BOCS as single indicators, instead of using subscale or item scores. We corrected for measurement error by fixing the error variances to values that were based on variances and the reliabilities of the scales. ${ }^{2}$ Reliability was based on Stratified Alpha, which is recommended for composite scales [58, 59]. Figure 2 shows a schematic picture of the LDS model.

Several hypotheses were examined using multivariate LDS models. For both the OBQ-CV and the CY-BOCS four time points were included: pre-treatment (T1), midtreatment (T2), post-treatment (T3), and follow-up (T4). We started with a Baseline Model in which latent changes between dysfunctional beliefs and OC symptoms were unrelated (see panel a in Fig. 3). When the Baseline Model is rejected, several hypotheses can be tested concerning the relations between changes in the both constructs. First, we tested whether changes in dysfunctional beliefs preceded changes in OCD severity (Lagged Effects Mediation Model). In this model, lagged effects between dysfunctional beliefs at time $\mathrm{T}$ on OCD severity at time $\mathrm{T}+1$ were specified (see panel b in Fig. 3). Second, we tested for the reversed effect: whether changes in dysfunctional beliefs at time $\mathrm{T}+1$ were the result of changes in OCD severity at time $\mathrm{T}$ (Lagged Effects Reversed Model; see panel c in

\footnotetext{
${ }^{2}$ Unique variances were calculated by $\operatorname{Var}(\mathrm{X}) *(1-$ reliability) [57].
} 
Fig. 2 Schematic representation of the LDS model

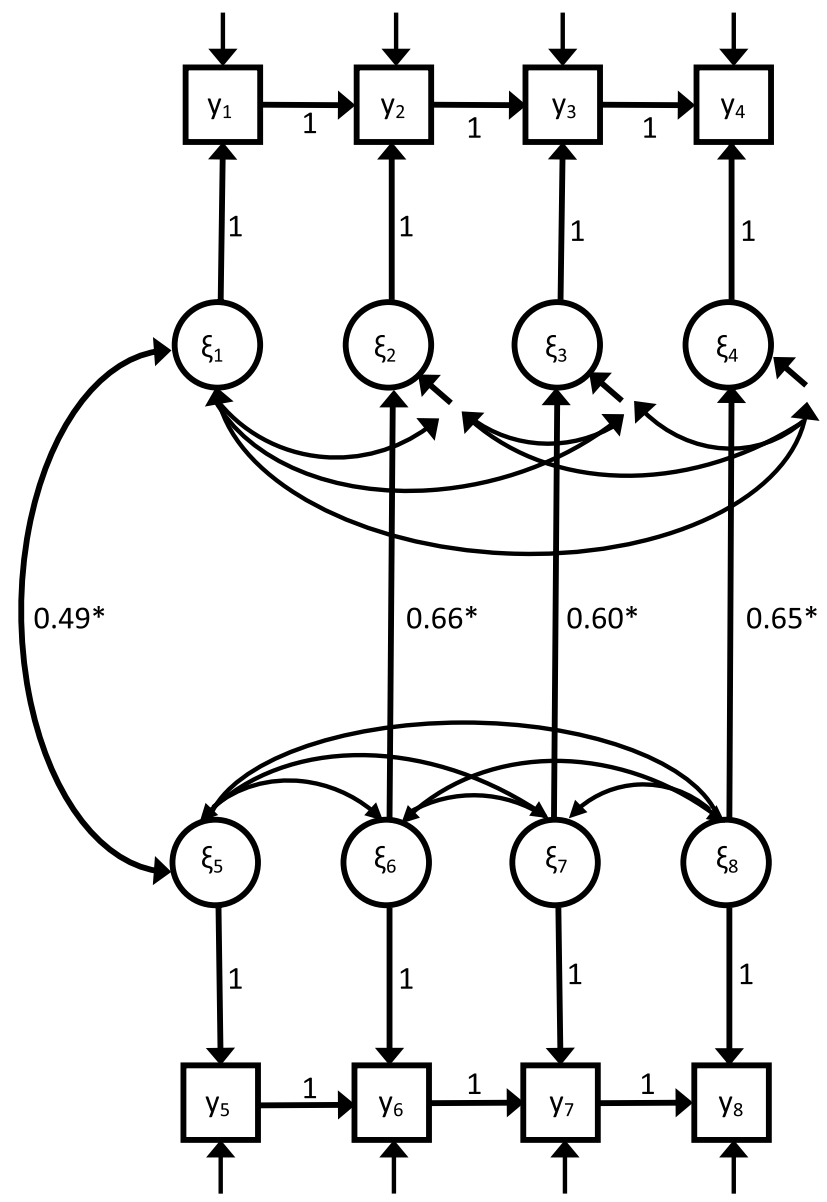

Index Figure 2

$\mathrm{Y}_{1}=\mathrm{OBQ} \mathrm{T} 1$

$\mathrm{Y}_{5}=\mathrm{CY}-\mathrm{BOCS} \mathrm{T} 1$

$\xi_{1}=\mathrm{Y}_{1}$

$\mathrm{Y}_{2}=\mathrm{OBQ} \mathrm{T} 2$

$\mathrm{Y}_{6}=\mathrm{CY}-\mathrm{BOCS} \mathrm{T} 2$

$\mathrm{Y}_{3}=\mathrm{OBQ} \mathrm{T} 3$

$\mathrm{Y}_{7}=\mathrm{CY}-\mathrm{BOCS} \mathrm{T} 3$

$\mathrm{Y}_{4}=\mathrm{OBQ} \mathrm{T} 4$

$\mathrm{Y}_{8}=\mathrm{CY}-\mathrm{BOCS} \mathrm{T} 4$
$\xi_{2}=\mathrm{Y}_{2}-\mathrm{Y}_{1}$

$\xi_{3}=\mathrm{Y}_{3}-\mathrm{Y}_{2}$

$\xi_{4}=\mathrm{Y}_{4}-\mathrm{Y}_{3}$

$\xi_{5}=\mathrm{Y}_{5}-\mathrm{Y}_{4}$

$\xi_{6}=\mathrm{Y}_{6}-\mathrm{Y}_{5}$

$\xi_{7}=\mathrm{Y}_{7}-\mathrm{Y}_{6}$

$\xi_{8}=\mathrm{Y}_{8}-\mathrm{Y}_{7}$

The value ' 1 ' indicates that paths were fixed at the value of ' 1 '.

Fig. 3). Third, because intervals between successive time points were relatively long (e.g., 8 weeks), we also tested a synchronous model. In this model, which is referred to as Synchronous Mediation Model, effects of changes in dysfunctional beliefs at time $\mathrm{T}$ on changes in OCD severity at time $\mathrm{T}$ were specified (see panel $\mathrm{d}$ in Fig. 3). This model implies that effects have occurred somewhere between time $\mathrm{T}$ and the previous assessment. The time interval between cause and effect is shorter than the interval between two successive measurement time-points [60]. Synchronous models provide weaker support for mediation than lagged models as the assumption of time precedence for cause-effect relations is not met. We also tested the reversed effect: whether changes in OCD severity predicted changes in beliefs within the same time interval (Synchronous Reversed Model; see panel e in Fig. 3). Finally, we tested whether there was a bidirectional relation between changes in beliefs and changes in OCD severity (Synchronous Reciprocal Model; see panel $\mathrm{f}$ in Fig. 3).

The following fit indices were selected to evaluate model fit: the minimum fit function Chi square statistic [61], the Akaike Information Criterion (AIC), the root mean square 
Fig. 3 Schematic representation of LDS models

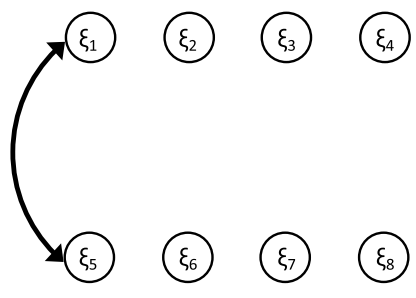

A Baseline Model
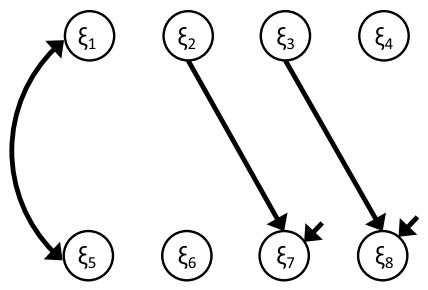

B Lagged Effects Mediation
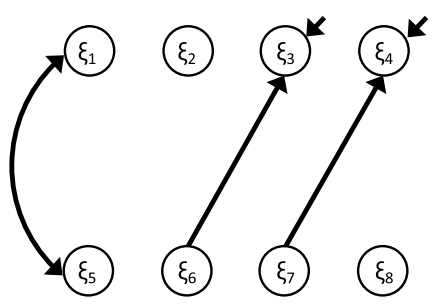

C Lagged Effects Reversed

Index Figure 3

$\xi_{1}=\mathrm{Y}_{1}$

$\xi_{2}=Y_{2}-Y_{1}$

$\xi_{3}=\mathrm{Y}_{3}-\mathrm{Y}_{2}$

$\xi_{4}=\mathrm{Y}_{4}-\mathrm{Y}_{3}$

$\xi_{5}=Y_{5}-Y_{4}$

$\xi_{6}=\mathrm{Y}_{6}-\mathrm{Y}_{5}$

$\xi_{7}=\mathrm{Y}_{7}-\mathrm{Y}_{6}$

$\xi_{8}=\mathrm{Y}_{8}-\mathrm{Y}_{7}$

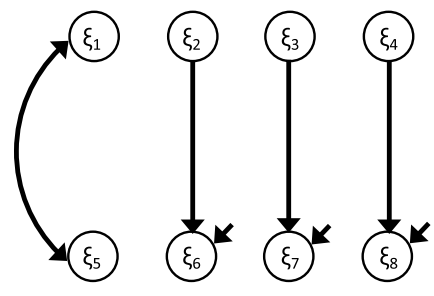

D Synchronous Mediation
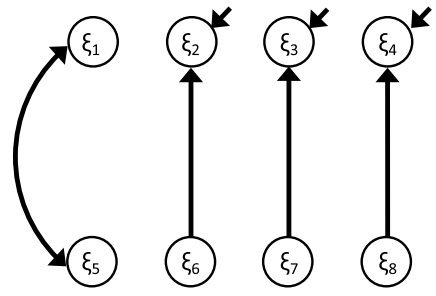

E Synchronous Reversed
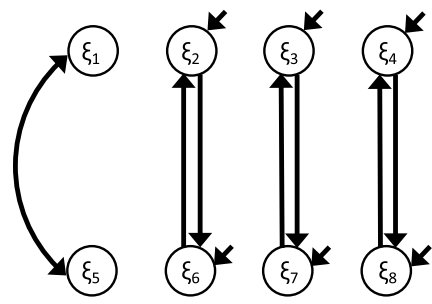

F Synchronous Reciprocal error of approximation (RMSEA), and the Comparative Fit Index (CFI). Low Chi square and AIC values, RMSEA values below 0.06 , and CFI values above 95 are generally assumed to indicate good model fit $[62,63]$. The Chi square difference test (the change of $\chi^{2}$ relative to the change in degrees of freedom) was used to test whether an alternative model leads to a significant improvement with regard to the original model [63].

\section{Sample Size}

There are no clear guidelines for the required sample size in structural equation modeling, as sample size requirements are affected by many factors, such as model complexity and estimation algorithm [63]. In the LDS model, most parameters are simple transformations of the covariances of the observed variables. The number of additional structural parameters that were estimated in the LDS model varied between 3 and 6 . Given the low number of structural parameters, the present sample size $(N=58)$ is considered acceptable.

\section{Results}

\section{Effect of Treatment}

To test whether the prerequisite of an effective treatment was met, we first examined whether there was a decrease 
Table 2 CY-BOCS: parameter estimates compared to baseline (T1)

\begin{tabular}{lllll}
\hline & $\mathrm{b}$ & $\mathrm{SE} \mathrm{b}$ & $95 \%$ CI & Pairwise comparisons \\
\hline T2 (8 sessions CBT) & -6.26 & 0.84 & $-7.94 ;-4.56$ & $\mathrm{~T} 2<\mathrm{T} 1(\mathrm{p}<0.001)$ \\
T3 (16 sessions CBT) & -12.92 & 1.18 & $-15.30 ;-10.54$ & $\mathrm{~T} 3<\mathrm{T} 2(\mathrm{p}<0.001)$ \\
T4 (16 weeks follow-up) & -14.67 & 1.00 & $-16.68 ;-12.65$ & $\mathrm{~T} 4<\mathrm{T} 3(\mathrm{p}=0.10)$ \\
\hline
\end{tabular}

\begin{tabular}{lrrllllllll}
\hline & $M$ & SD & \multicolumn{2}{l}{ Pearson correlation $(r)$} \\
\cline { 3 - 10 } & & & CB 1 & CB 2 & CB 3 & CB 4 & OBQ 1 & OBQ 2 & OBQ 3 & OBQ 4 \\
\hline CB 1 & 24.71 & 5.04 & & & & & & & & \\
CB 2 & 18.43 & 5.93 & $0.41^{* *}$ & & & & & & \\
CB 3 & 11.90 & 8.86 & $0.51^{* *}$ & $0.76^{* *}$ & & & & & \\
CB 4 & 10.10 & 6.43 & $0.41^{* *}$ & $0.68^{* *}$ & $0.72^{* *}$ & & & & \\
OBQ 1 & 107.76 & 29.85 & $0.44^{* *}$ & $0.38^{* *}$ & $.25^{\mathrm{t}}$ & $0.30^{*}$ & & & \\
OBQ 2 & 100.76 & 33.65 & $0.33^{*}$ & $0.52^{* *}$ & $38^{* *}$ & $0.37^{* *}$ & $0.88^{* *}$ & & & \\
OBQ 3 & 91.90 & 34.46 & $0.45^{* *}$ & $0.63^{* *}$ & $0.62^{* *}$ & $0.50^{* *}$ & $0.75^{* *}$ & $0.85^{* *}$ & & \\
OBQ 4 & 93.40 & 30.99 & $0.35^{* *}$ & $0.53^{* *}$ & $0.41^{* *}$ & $0.44^{* *}$ & $0.79^{* *}$ & $0.87^{* *}$ & $0.89 * *$ & \\
\hline
\end{tabular}

$\mathrm{CB}=\mathrm{CY}-\mathrm{BOCS}, 1=\mathrm{T} 1,2=\mathrm{T} 2,3=\mathrm{T} 3,4=\mathrm{T} 4$

*Correlation is significant at the 0.05 level, **Correlation is significant at the 0.01 level
Table 3 Means, standard deviations and correlations between CY-BOCS and OBQ-CV scores change in beliefs was the result of a change in OCD severity also showed inadequate fit indices. The inadequate fit of the lagged effects models could be explained by the relatively long time interval between assessments. Therefore, we estimated a series of synchronous models which allow shorter time intervals between cause and effect. The synchronous models (Models 4, 5) fitted the data significantly better than the Baseline Model (indicated by significant Chi square difference tests), and showed acceptable fit indices. Best model fit was found for the Synchronous Reversed Model (Model 5) which showed excellent fit values on all goodness of fit measures (low and non-significant Chi square value, RMSEA $<0.05$, and CFI $>0.95$ ), and the lowest value of the AIC. The Synchronous Reciprocal Model (Model 6) is nested in the Synchronous Reversed Model. Model 6 did not show significant improvement to Model 5 as indicated by the non-significant Chi square difference test, and three path coefficients were non-significant (all reflecting an effect of the OBQ on the CY-BOCS). Therefore, this model was rejected.

The best fitting model was the Synchronous Reversed Model. Results for this model showed significant, positive effects of the CY-BOCS on the OBQ at each assessment point. Parameter estimates were 0.18 (SD 0.04) for $\Delta$ CY-BOCS ${ }_{\mathrm{T} 1-\mathrm{T} 2} \rightarrow \Delta \mathrm{OBQ}_{\mathrm{T} 1-\mathrm{T} 2} ; 0.20$ (SD 0.04) for $\Delta \mathrm{CY}-\mathrm{BOCS}_{\mathrm{T} 2-\mathrm{T} 3} \rightarrow \Delta \mathrm{OBQ}_{\mathrm{T} 2-\mathrm{T} 3}$; and 0.16 (SD 0.03) for $\Delta \mathrm{CY}-\mathrm{BOCS}_{\mathrm{T} 3-\mathrm{T} 4} \rightarrow \Delta \mathrm{OBQ}_{\mathrm{T} 3-\mathrm{T} 4}$. Squared multiple correlations were 0.43 ; 0.36 ; and 0.42 respectively (see Fig. 2 for this model). 
Table 4 Fit indices

\begin{tabular}{|c|c|c|c|c|c|c|c|}
\hline Model & $\begin{array}{l}\chi^{2} \\
(p \text { value })\end{array}$ & $\mathrm{df}$ & RMSEA & 90\% CI RMSEA & $\begin{array}{l}\text { close fit RMSEA } \\
p \text { value }\end{array}$ & AIC & $\mathrm{CFI}$ \\
\hline (1) Baseline model & $\begin{array}{l}54.38 \\
(<0.001)\end{array}$ & 15 & 0.18 & $0.12-0.24$ & 0.001 & 84.37 & 0.92 \\
\hline (2) Lagged effects mediation & $\begin{array}{l}52.81 \\
(<0.001)\end{array}$ & 13 & 0.20 & $0.14-0.27$ & $<0.001$ & 88.75 & 0.92 \\
\hline Difference model 2 versus model 1 & $\begin{array}{l}1.57 \\
(0.46)\end{array}$ & 2 & & & & & \\
\hline (3) Lagged Effects Reversed & $\begin{array}{l}48.85 \\
(<0.001)\end{array}$ & 13 & 0.19 & $0.13-0.26$ & $<0.001$ & 86.80 & 0.93 \\
\hline Difference model 3 versus model 1 & $\begin{array}{l}5.53 \\
(0.06)\end{array}$ & 2 & & & & & \\
\hline (4) Synchronous mediation & $\begin{array}{l}20.77 \\
(0.05)\end{array}$ & 12 & 0.11 & $0.00-0.19$ & 0.14 & 67.72 & 0.98 \\
\hline Difference model 4 versus model 1 & $\begin{array}{l}33.61 \\
(<0.001)\end{array}$ & 3 & & & & & \\
\hline (5) Synchronous reversed & $\begin{array}{l}9.02 \\
(0.70)\end{array}$ & 12 & 0.00 & $0.00-0.10$ & 0.81 & 56.75 & 1.00 \\
\hline Difference model 5 versus model 1 & $\begin{array}{l}45.35 \\
(<0.001)\end{array}$ & 3 & & & & & \\
\hline (6) Synchronous reciprocal & $\begin{array}{l}4.19 \\
(0.90)\end{array}$ & 9 & 0.00 & $0.00-0.06$ & 0.93 & 58.17 & 1.00 \\
\hline Difference model 6 versus model 5 & $\begin{array}{l}4.83 \\
(0.19)\end{array}$ & 3 & & & & & \\
\hline
\end{tabular}

\section{Discussion}

The aim of the present study was to examine if changing dysfunctional beliefs was a mediator of CBT for pediatric OCD. Fifty-eight children with OCD (8-18 years old) received sixteen weekly sessions of CBT consisting of ERP and CT. Dysfunctional beliefs and OCD severity were assessed pre-treatment, mid-treatment, post-treatment, and at 16-week follow-up. According to cognitive models, we expected cognitive changes to precede changes in OCD severity. Contrary to this hypothesis, results showed that changes in OCD severity statistically predicted changes in dysfunctional beliefs rather than the reverse. In other words, changes in severity explained changes in dysfunctional beliefs within time intervals. More specifically, changes in OCD severity explained $43 \%$ of the change in beliefs at mid-treatment, $36 \%$ between mid- and posttreatment, and $42 \%$ between post-treatment and follow-up. It is important to note that the present results do not allow for conclusions about causality, because we did not find a relation between cognitions and OCD severity over time (across assessment points). Therefore, we cannot determine whether a decrease in dysfunctional beliefs actually was an effect of a decrease in OCD severity. Nevertheless, the present findings cast doubt on the assumption of cognitive models suggesting that changing beliefs plays a key role in the treatment of OCD.
In line with the present results, several previous studies evaluating mediating mechanisms in psychological treatment for OCD were not supportive for cognitive models [34, 41-44]. However, this field of research is still in its infancy and is hampered by the limited amount of studies, the main focus on adult samples, and methodological limitations and differences across studies. There are some other issues that merit discussion.

Studying cognitive mediation is a challenging task. One of the biggest challenges is to measure actual thought processes. Cognitive processes may rely on conscious thoughts as well as on unconscious, automatic thoughts. Explicit measures, either standardized or idiographic, can only provide indications of beliefs that are accessible for personal introspection. In addition to explicit measures, implicit paradigms may be needed to shed light on the role of unconscious, automatic thoughts $[64,65]$. Although still in its early stages, several cognitive bias paradigms have been developed for this purpose [66]. It would be interesting to use such paradigms in future studies. It could be for example, that contrary to the explicit approach in cognitive therapy, ERP addresses implicit cognitive processes. Implicit paradigms may be more suitable to detect these mechanisms.

Another challenge is that mediation requires a relation between the mediating variable and the outcome variable over time [37]. One could wonder if-even in case of established cognitive mediation-the mediating processes can 
be disentangled over time. One may assume that as soon as thought processes change, for example the patient does not overestimate the importance of an intrusion anymore, no raise in anxiety will appear, and consequently there may be no urge to perform compulsions. These processes may occur in acute response to each other, making it impossible to observe a temporal lag between a change in cognitive processes and in OC symptoms [67]. Following this line of reasoning, it may be complicated to get a grip on these fast and dynamic processes.

A third challenge is that mediating processes may differ across individuals. This hypothesis is supported by a study in adult OCD patients on the role of dysfunctional beliefs in CBT [42]. Indeed, there is some evidence that not all OCD patients experience more dysfunctional beliefs than nonclinical individuals [68, 69], and cognitive mediation may not be expected for these patients. In addition, our findings showed a wide range of OBQ-CV scores, indicating that there are substantial individual differences in dysfunctional beliefs, and consequently there may be differences in cognitive change processes during treatment.

Although the present study has several strengths such as a longitudinal design with a mid-treatment assessment, a representative sample of youth with OCD, and the use of a treatment protocol that has already been implemented in clinical practice, the study has some limitations too. First, the present sample size did not allow for adding extra variables to the models, and therefore we could not control for effects of possible moderating variables such as OCD subtype or developmental level. However, a previous study showed no effect of age on OBQ-CV score in a clinical OCD sample (mainly the same sample as the present study) [28]. This finding makes it less likely that results would have been different when age was included. Furthermore, the present treatment protocol allowed for tailoring cognitive interventions to the individual to preclude the risk that these interventions would have been too difficult to understand for younger children, or too childish for older children. This way, we aimed to provide a treatment that suited all participants. Second, due to the time interval between assessment points we may have been unable to detect change processes that have occurred in-between assessment points. Third, reports of dysfunctional beliefs were solely based on a standardized questionnaire. The OBQ-CV was selected because adequate reliability and validity had been demonstrated in pediatric OCD and in a youth community sample [28, 70]. Furthermore, the OBQ-CV includes multiple dysfunctional belief domains assumed to be relevant in OCD, instead of a limited selection of domains [71, 72]. However, despite these strong features of the OBQ-CV, the incorporation of multiple belief domains also entails a disadvantage. Participants who frequently experience beliefs in one domain, may have a relatively low OBQ-CV total score despite the high frequency of particular beliefs. In these cases only small changes can be found, and even if dysfunctional beliefs change during treatment it would be hard to demonstrate mediation effects over time. We have considered to perform analyses using OBQ subscales instead of a general total scale. However, besides that this would have added extra parameters to the model, a study on psychometric properties of the OBQ-CV showed high correlations among subscales, and a single higher-order factor (OBQ total score) that explained the correlations between the subscales quite well [28]. For these reasons, we did not differentiate between subscales. Another complicating factor of using explicit measures is that a certain level of insight in thought processes is required for a valid report of dysfunctional beliefs. This may be demanding for all individuals, and especially for children as meta-cognitive skills are not fully developed at this age. Finally, the main part of the sample was of a Western background. This may limit the generalizability of the results to samples of other cultural backgrounds.

Notwithstanding these limitations, some clinical implications and recommendations for future research can be derived from the present study. Based on the present results, we conclude that restructuring explicit cognitions may not be a necessary component in the treatment of OCD in children and adolescents. At least not for all patients with OCD. For future studies it would be interesting to shed more light on potential moderating variables, for example OCD subtype and children with and without obsessions (tic-related OCD), in combination with mediating variables. Furthermore, idiosyncratic and implicit measures could be combined with standardized, explicit measures of dysfunctional beliefs to examine the role of both explicit and implicit cognitive processes in CBT.

For therapists it would be interesting to know if treatment for pediatric OCD could be confined to solely ERP. The present results do not allow for such a conclusion. First of all, our results need to be replicated. Second, this conclusion would assume a single, one-to-one relationship between $\mathrm{CT}$ and a change in dysfunctional beliefs. This seems no realistic representation of matters. We cannot equate CT with cognitive change, and ERP with behavioral change. At present, the actual mechanisms of change of CT and ERP are inadequately understood. Different explicit and implicit processes may be active in CT and ERP, and there may be different ways to achieve treatment effect. Furthermore, in many cases elements of exposure are incorporated in CT in the form of behavioral experiments, and ERP is often complemented by cognitive interventions. The latter is explicitly prescribed in the inhibitory learning model of ERP where expectations about what may happen following exposure are challenged by the therapist [12]. Challenging cognitions is not specifically prescribed in the habituation model of ERP, but this intervention can be used to support ERP [13]. 
Future studies that aim to evaluate mediating (cognitive) mechanisms in treatment for OCD, should include more in-treatment assessment points. To gain more insight in change processes of dysfunctional beliefs and OC symptoms, these constructs could be measured each session or every day during treatment. Case-based timeseries designs can then be used to closely follow changes over time within individuals $[42,45,46]$, before resource demanding, large randomized controlled trials are conducted. Although seldom used, case-based time series designs are recognized as fair methodological approaches for treatment studies [73], and can provide information about what interventions work for whom. The latter may be especially important in the light of the large individual differences in treatment effects for pediatric OCD, and because mediating mechanisms may differ across patients.

\section{Summary}

It is generally assumed that explicitly challenging dysfunctional cognitions plays an important role in psychological treatment for OCD. However, to date there hardly is any empirical support for this assumption, especially for pediatric OCD. The aim of the present study was to examine if changing dysfunctional beliefs is a mediator of CBT for pediatric OCD. Fifty-eight children and adolescents (8-18 years; 24 boys) with a primary diagnosis of OCD received sixteen weekly sessions manualized CBT. CBT included exposure and response prevention, and cognitive interventions. Dysfunctional beliefs (OBQ-CV) and OCD severity (CY-BOCS) were measured at different time-points during treatment (pre-treatment, mid-treatment, post-treatment), and at 16-week follow-up. Results showed that both OCD severity and dysfunctional beliefs decreased during CBT. However, contrary to what would be expected from cognitive models, changes in severity statistically predicted changes in dysfunctional beliefs within the same time interval, rather than the reverse. The present results did not support the hypothesis that changing dysfunctional beliefs is a mediating mechanism in CBT for childhood OCD. This finding suggests that restructuring explicit cognitions might not be a necessary component in the treatment of OCD in children and adolescents. Future studies are needed to replicate the present findings and shed more light on the role of explicit and implicit cognitions in CBT for childhood OCD.

Acknowledgements We thank the therapists, the children and their parents for their participation in this study.

\section{Compliance with Ethical Standards}

Conflict of interest The authors declare that they have no conflict of interest.

Open Access This article is distributed under the terms of the Creative Commons Attribution 4.0 International License (http://creativeco mmons.org/licenses/by/4.0/), which permits unrestricted use, distribution, and reproduction in any medium, provided you give appropriate credit to the original author(s) and the source, provide a link to the Creative Commons license, and indicate if changes were made.

\section{References}

1. Franklin ME, Kratz HE, Freeman JB, Ivarsson T, Heyman I, Sookman D et al (2015) Cognitive-behavioral therapy for pediatric obsessive-compulsive disorder: empirical review and clinical recommendations. Psychiatry Res 227:78-92

2. O'Kearney RT, Anstey K, Von Sanden C, Hunt A (2010) Behavioural and cognitive behavioural therapy for obsessive compulsive disorder in children and adolescents (Review). Cochrane Database Syst Rev 2010(4): 1-50

3. Sanchez-Meca J, Rosa-Alcazar AI, Iniesta-Sepulveda M, RosaAlcazar A (2014) Differential efficacy of cognitive-behavioral therapy and pharmacological treatments for pediatric obsessivecompulsive disorder: a meta-analysis. J Anxiety Disord 28:31-44

4. Skarphedinsson G, Hanssen-Bauer K, Kornør H, Heiervang ER, Landrø NI, Axelsdottir B et al (2014) Standard individual cognitive behaviour therapy for paediatric obsessive-compulsive disorder: a systematic review of effect estimates across comparisons. Nord J Psychiatry 69:81-92

5. Watson HJ, Rees CS (2008) Meta-analysis of randomized, controlled treatment trials for pediatric obsessive-compulsive disorder. J Child Psychol Psychiatry 49:489-498

6. The Pediatric OCD Treatment Study (POTS) Team (2004) Cognitive-behavior therapy, sertraline, and their combination for children and adolescents with obsessive-compulsive disorder-The Pediatric OCD Treatment Study (POTS) randomized controlled trial. JAMA 292:1969-1976

7. Torp NC, Dahl K, Skarphedinsson G, Thomsen PH, Valderhaug $R$, Weidle B et al (2015) Effectiveness of cognitive behavior treatment for pediatric obsessive-compulsive disorder: acute outcomes from the Nordic Long-term OCD Treatment Study (NordLOTS). Behav Res Ther 64:15-23

8. Wolters LH, de Haan E, Hogendoorn SM, Boer F, Prins PJM (2016) Severe pediatric obsessive compulsive disorder and comorbid autistic symptoms: effectiveness of cognitive behavioral therapy. J Obsessive Compuls Relat Disord 10:69-77

9. Ale CM, McCarthy DM, Rothschild LM, Whiteside SP (2015) Components of cognitive behavioral therapy related to outcome in childhood anxiety disorders. Clin Child Fam Psychol Rev 18:240-251

10. Craske MG, Treanor M, Conway CC, Zbozinek T, Vervliet B (2014) Maximizing exposure therapy: an inhibitory learning approach. Behav Res Ther 58:10-23

11. Himle MB (2015) Let truth be thy aim, not victory: comment on theory-based exposure process. J Obsessive Compuls Relat Disord 6:183-190

12. Arch JJ, Abramowitz JS (2015) Exposure therapy for obsessivecompulsive disorder: an optimizing inhibitory learning approach. J Obsessive Compuls Relat Disord 6:174-182 
13. Benito KG, Walther M (2015) Therapeutic process during exposure: habituation model. J Obsessive Compuls Relat Disord 6:147-157

14. Berman NC, Fang A, Hansen N, Wilhelm S (2015) Cognitivebased therapy for OCD: role of behavior experiments and exposure processes. J Obsessive Compuls Relat Disord 6:158-166

15. Salkovskis PM (1985) Obsessional-compulsive problems-a cognitive-behavioral analysis. Behav Res Ther 23:571-583

16. Bolton D, Dearsley P, Madronal-Luque R, Baron-Cohen S (2002) Magical thinking in childhood and adolescence: development and relation to obsessive compulsion. Br J Dev Psychol 20:479-494

17. Evans DW, Milanak ME, Medeiros B, Ross JL (2002) Magical beliefs and rituals in young children. Child Psychiatry Hum Dev 33:43-58

18. Farrell L, Barret P (2006) Obsessive-compulsive disorder across developmental trajectory: cognitive processing of threat in children, adolescents and adults. Br J Psychol 97:95-114

19. Libby S, Reynolds S, Derisley J, Clark S (2004) Cognitive appraisals in young people with obsessive-compulsive disorder. J Child Psychol Psychiatry 45:1076-1084

20. Magnúsdóttir I, Smári J (2004) Are responsibility attitudes related to obsessive-compulsive symptoms in children? Cogn Behav Ther $33: 21-26$

21. Mather A, Cartwright-Hatton S (2004) Cognitive predictors of obsessive-compulsive symptoms in adolescence: a preliminary investigation. J Clin Child Adolesc Psychol 33:743-749

22. Matthews L, Reynolds S, Derisley J (2007) Examining cognitive models of obsessive compulsive disorder in adolescents. Behav Cogn Psychother 35:149-163

23. Muris P, Meesters C, Rassin E, Merckelbach H, Campbell J (2001) Thought-action fusion and anxiety disorders symptoms in normal adolescents. Behav Res Ther 39:843-852

24. Reeves J, Reynolds S, Coker S, Wilson C (2010) An experimental manipulation of responsibility in children: a test of the inflated responsibility model of obsessive-compulsive disorder. J Behav Ther Exp Psychiatry 41:228-233

25. Ye HJ, Rice KG, Storch EA (2008) Perfectionism and peer relations among children with obsessive-compulsive disorder. Child Psychiatry Hum Dev 39:415-426

26. Barrett P, Healy-Farrell L (2003) Perceived responsibility in juvenile obsessive-compulsive disorder: an experimental manipulation. J Clin Child Adolesc Psychol 32:430-441

27. Verhaak LM, De Haan E (2007) Cognitions in children with OCD-A pilot study for age specific relations with severity. Eur Child Adolesc Psychiatry 16:353-361

28. Wolters LH, Hogendoorn SM, Koolstra T, Vervoort L, Boer F, Prins PJM et al (2011) Psychometric properties of a Dutch version of the Obsessive Beliefs Questionnaire-Child Version (OBQ-CV). J Anxiety Disord 25:714-721

29. Wolters LH, Hogendoorn SM, Oudega M, Vervoort L, De Haan E, Prins PJM et al (2012) Psychometric properties of the Dutch version of the Meta-Cognitions Questionnaire-Adolescent Version (MCQ-A) in non-clinical adolescents and adolescents with obsessive-compulsive disorder. J Anxiety Disord 26:343-351

30. Barrett PM, Farrell L, Pina AA, Peris TS, Piacentini J (2008) Evidence-based psychosocial treatments for child and adolescent obsessive-compulsive disorder. J Clin Child Adolesc Psychol 37:131-155

31. March JS, Mulle K (1998) OCD in children and adolescents: a cognitive-behavioral treatment manual. Guilford, New York

32. Williams TI, Salkovskis PM, Forrester L, Turner S, White H, Allsopp MA (2010) A randomized controlled trial of cognitive behavioural treatment for obsessive compulsive disorder in children and adolescents. Eur Child Adolesc Psychiatry 19:449-456

33. Olatunji BO, Davis ML, Powers MB, Smits JAJ (2013) Cognitive-behavioral therapy for obsessive-compulsive disorder: a meta-analysis of treatment outcome and moderators. J Psychiatr Res 47:33-41

34. Olatunji BO, Rosenfield D, Tart CD, Cottraux J, Powers MB, Smits JA (2013) Behavioral versus cognitive treatment of obsessive-compulsive disorder: an examination of outcome and mediators of change. J Consult Clin Psychol 81:415-428

35. Cougl JR, Lee HJ (2014) Pathological and non-pathological features of obsessive-compulsive disorder: revisiting basic assumptions of cognitive models. J Obsessive Compuls Relat Disord 3:12-20

36. Maric M, Wiers RW, Prins PJ (2012) Ten ways to improve the use of statistical mediation analysis in the practice of child and adolescent treatment research. Clin Child Fam Psychol Rev 15: 177-191

37. Maric M, Prins PJM, Ollendick TH (eds) (2015) Moderators and mediators of youth treatment outcomes. Oxford University Press: New York

38. Williams TI, Salkovskis PM, Forrester EA, Allsopp MA (2002) Changes in symptoms of OCD and appraisal of responsibility during cognitive behavioural treatment: a pilot study. Behav Cogn Psychother 30: 69-78

39. Overton SM, Menzies RG (2012) Cognitive change during treatment of compulsive checking. Behav Change 22:172-184

40. Wilhelm S, Berman NC, Keshaviah A, Schwartz RA (2015) Mechanisms of change in cognitive therapy for obsessive compulsive disorder: role of maladaptive beliefs and schemas. Behav Res Ther 65:5-10

41. Anholt GE, Kempe P, De Haan E, van Oppen P, Cath DC, Smit $\mathrm{JH}$ et al (2008) Cognitive versus Behavior therapy: processes of change in the treatment of obsessive-compulsive disorder. Psychother Psychosom 77:38-42

42. Polman A, Bouman TK, Van Geert PLC, de Jong PJ, Den Boer JA (2011) Dysfunctional beliefs in the process of change of cognitive treatment in obsessive compulsive checkers. Clin Psychol Psychother 18:256-273

43. Su Y-J, Carpenter JK, Zandberg LJ, Simpson HB, Foa EB (2016) Cognitive mediation of symptom change in exposure and response prevention for obsessive-compulsive disorder. Behav Ther 47:474-486

44. Woody SR, Whittal ML, McLean PD (2011) Mechanisms of symptom reduction in treatment for obsessions. J Consult Clin Psychol 79:653-664

45. Rheaume J, Ladouceur R (2000) Cognitive and behavioural treatments of checking behaviours: an examination of individual cognitive change. Clin Psychol Psychother 7:118-127

46. Storchheim LE, O'Mahony JE (2006) Compulsive behaviours and levels of belief in obsessive-compulsive disorder: a caseseries analysis of their interrelationships. Clin Psychol Psychother 13:64-79

47. De Haan E, Wolters LH (2009) Behandeling van de dwangstoornis bij kinderen en adolescenten. Met het cognitiefgedragstherapeutisch protocol Bedwing je dwang. [Treatment of OCD in children and adolescents. With the cognitive behavioural treatment protocol Control your OCD.]. Bohn Stafleu van Loghum, Houten, NL

48. Silverman WK, Albano AM (1996) Anxiety disorders interview schedule for DSM-IV child version, child interview schedule. The Psychological Corporation, San Antonio

49. Silverman WK, Albano AM (1996) Anxiety disorders interview schedule for DSM-IV child version, parent interview schedule. The Psychological Corporation, San Antonio

50. Silverman WK, Saavedra LM, Pina AA (2001) Test-retest reliability of anxiety symptoms and diagnoses with the anxiety disorders interview schedule for DSM-IV: child and parent versions. J Am Acad Child Adolesc Psychiatry 40:937-944 
51. Wood JJ, Piacentini JC, Bergman RL, McCracken J, Barrios V (2002) Concurrent validity of the anxiety disorders section of the anxiety disorders interview schedule for DSM-IV: child and parent versions. J Clin Child Adolesc Psychol 31:335-342

52. Scahill L, Riddle MA, McSwigginHardin M, Ort SI, King RA, Goodman WK et al (1997) Children's Yale-Brown obsessive compulsive scale: reliability and validity. J Am Acad Child Adolesc Psychiatry 36:844-852

53. Steyer R, Eid M, Schwenkmezger P (1997) Modeling true intraindividual change: true change as a latent variable. Methods Psychol Res Online 2:21-33

54. Selig JP, Preacher KJ (2009) Mediation models for longitudinal data in developmental research. Res Hum Dev 6:144-164

55. McArdle JJ, Hamagami F (2001) Latent difference score structural models for linear dynamic analyses with incomplete longitudinal data. In: Collins LSA (ed) Methods for the analysis of change. American Psychological Association, Washington D.C, pp 13-175

56. Ferrer E, McArdle JJ (2003) Alternative structural models for multivariate longitudinal data analysis. Struct Equ Modeling 10:493-524

57. Bollen KA (1989) Structural equations with latent variables. Wiley, New York

58. Nunnally J, Bernstein I (1994) Psychometric theory, 3rd edn. McGraw-Hill, New York

59. Osburn HG (2000) Coefficient alpha and related internal consistency reliability coefficients. Psychol Methods 5:343-355

60. Dwyer JH (1983) Statistical models for the social and behavioral sciences. Oxford University Press, New York

61. Olsson UH, Foss T, Breivik E (2004) Two equivalent discrepancy functions for maximum likelihood estimation: do their test statistics follow a non-central Chi-square distribution under model misspecification? Sociol Methods Res 32:453-500

62. Hu L, Bentler PM (1999) Cutoff criteria for fit indexes in covariance structure analysis: conventional criteria versus new alternatives. Struct Equ Modeling 6:1-55
63. Kline RB (2005) Principles and practice of structural equation modeling, 2 edn. The Guilford Press, New York

64. Fazio RH, Olson MA (2003) Implicit measures in social cognition research: their meaning and use. Annu Rev Psychol 54:297-327

65. MacLeod C, Cohen IL (1993) Anxiety and the interpretation of ambiguity: a text comprehension study. J Abnorm Psychol 102:238-247

66. Koster EHW, Bernstein A (2015) Introduction to the special issue on Cognitive bias modification: taking a step back to move forward? J Behav Ther Exp Psychiatry 49: 1-4

67. Hollon SD, DeRubeis RJ (2009) Mediating the effects of cognitive therapy for depression. Cogn Behav Ther 38:43-47

68. Calamari JE, Cohen RJ, Rector NA, Szacun-Shimizu K, Riemann BC, Norberg MM (2006) Dysfunctional belief-based obsessivecompulsive disorder subgroups. Behav Res Ther 44:1347-1360

69. Taylor S, Abramowitz JS, Mckay D, Calamari JE, Sookman D, Kyrios M et al (2006) Do dysfunctional beliefs play a role in all types of obsessive-compulsive disorder? J Anxiety Disord 20:85-97

70. Coles ME, Wolters LH, Sochting I, De Haan E, Pietrefesa AS, Whiteside SP (2010) Development and initial validation of the Obsessive Belief Questionnaire-Child Version (OBQ-CV). Depress Anxiety 27:982-991

71. Obsessive Compulsive Cognitions Working Group (OCCWG) (1997) Cognitive assessment of obsessive-compulsive disorder. Behav Res Ther 35:667-681

72. Obsessive Compulsive Cognitions Working Group (OCCWG) (2001) Development and initial validation of the obsessive beliefs questionnaire and the interpretation of intrusions inventory. Behav Res Ther 39:987-1006

73. Borckardt JJ, Nash MR, Murphy MD, Moore M, Shaw D, O'Neil $P$ (2008) Clinical practice as natural laboratory for psychotherapy research-A guide to case-based time-series analysis. Am Psychol 63:77-95 